\title{
Correction to: Hypoglycemia, Malnutrition and Body Composition
}

I. Khanimov, M. Shimonov, J. Wainstein, and Eyal Leibovitz

\section{Correction to:}

Adv Exp Med Biol - Advances in Internal Medicine https://doi.org/10.1007/5584_2020_526

The original version of this chapter was inadvertently published with a subtitle which was a duplication of the chapter title. The chapter subtitle has been removed.

The original article was corrected. 